\title{
Article
}

\section{The relative efficiencies of bars and clumps in driving disc stars to retrograde motion}

Fiteni, Karl, Caruana, Joseph, Amarante, Joao A.S., Debattista, Victor $\mathrm{P}$ and Beraldo E silva, Leandro

Available at http://clok.uclan.ac.uk/37109/

Fiteni, Karl, Caruana, Joseph, Amarante, Joao A.S., Debattista, Victor P ORCID: 0000-0001-7902-0116 and Beraldo E silva, Leandro ORCID: 0000-0002-07401507 (2021) The relative efficiencies of bars and clumps in driving disc stars to retrograde motion. Monthly Notices of the Royal Astronomical Society, 503 (1). pp. 1418-1430. ISSN 0035-8711

It is advisable to refer to the publisher's version if you intend to cite from the work. http://dx.doi.org/10.1093/mnras/stab619

For more information about UCLan's research in this area go to http://www.uclan.ac.uk/researchgroups/ and search for <name of research Group>.

For information about Research generally at UCLan please go to http://www.uclan.ac.uk/research/

All outputs in CLoK are protected by Intellectual Property Rights law, including Copyright law. Copyright, IPR and Moral Rights for the works on this site are retained by the individual authors and/or other copyright owners. Terms and conditions for use of this material are defined in the policies page.

\section{CLoK}

Central Lancashire online Knowledge www.clok.uclan.ac.uk

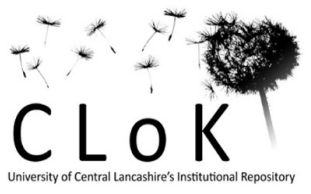




\title{
The relative efficiencies of bars and clumps in driving disc stars to retrograde motion
}

\author{
Karl Fiteni $^{\circledR}$, ${ }^{1 \star}$ Joseph Caruana, $^{1,2}$ João A. S. Amarante ${ }^{\circledR},{ }^{3,4}$ Victor P. Debattista $^{(1,5}$ \\ and Leandro Beraldo e Silva ${ }^{\oplus 5}$ \\ ${ }^{1}$ Institute of Space Sciences \& Astronomy, University of Malta, Msida MSD 2080, Malta \\ ${ }^{2}$ Department of Physics, University of Malta, Msida MSD 2080, Malta \\ ${ }^{3}$ Key Laboratory for Research in Galaxies and Cosmology, Shanghai Astronomical Observatory, Chinese Academy of Sciences, 80 Nandan Road, Shanghai \\ 200030, China \\ ${ }^{4}$ University of Chinese Academy of Sciences, No.19A Yuquan Road, Beijing 100049, China \\ ${ }^{5}$ Jeremiah Horrocks Institute, University of Central Lancashire, Preston PR1 2HE, UK
}

Accepted 2021 February 28. Received 2021 February 24; in original form 2020 December 22

\begin{abstract}
The presence of stars on retrograde orbits in disc galaxies is usually attributed to accretion events, both via direct accretion, and through the heating of the disc stars. Recent studies have shown that retrograde orbits can also be produced via scattering by dense clumps, which are often present in the early stages of a galaxy's evolution. However, so far it has been unclear whether other internally driven mechanisms, such as bars, are also capable of driving retrograde motion. Therefore, in this paper, we investigate the efficiencies with which bars and clumps produce retrograde orbits in disc galaxies. We do this by comparing the retrograde fractions and the spatial distributions of the retrograde populations in four $\mathrm{N}$-body + smooth particle hydrodynamics (SPH) simulations of isolated disc galaxies spanning a range of evolutionary behaviours. We find that both bars and clumps are capable of generating significant retrograde populations of order $\sim 10$ per cent of all stars. We also find that while clump-driven retrograde stars may be found at large galactocentric radii, bar-driven retrograde stars remain in the vicinity of the bar, even if the bar dissolves. Consequently, we find that retrograde stars in the Solar neighbourhood in the clumpy models are exclusively clump-driven, but this is a trace population, constituting $0.01-0.04$ per cent of the total stellar population in this region. Finally, we find that neither bars (including dissolving ones) nor clumps in the models are able to produce rotationally supported counterrotating discs.
\end{abstract}

Key words: galaxies: bar-galaxies: disc-galaxies: evolution-galaxies: kinematics and dynamics.

\section{INTRODUCTION}

According to the prevailing lambda cold dark matter $(\Lambda \mathrm{CDM})$ model, the early evolutionary history of disc galaxies was dominated by hierarchical merging and accretion of material (White \& Frenk 1991). However, the subsequent decrease in the frequency of major mergers with time meant that secular, internally driven, processes were able to drive most of the later evolution.

Bars are one of the main drivers of secular evolution in disc galaxies (Athanassoula 2013), and previous studies have shown that they can significantly impact their host galaxy through a number of mechanisms (see reviews by Kormendy \& Kennicutt 2004; Kormendy 2013). For example, it is well established that bars facilitate the exchange of angular momentum, both throughout the disc, and between different stellar components (Hohl 1978; Sellwood 1980). Angular momentum exchange with the dark matter halo via dynamical friction has also been shown to cause bars to slow down and grow radially (Weinberg 1985; Hernquist \& Weinberg 1992; Debattista \& Sellwood 2000; Valenzuela \& Klypin 2003; Martinez-

^E-mail: karl.fiteni.12@um.edu.mt
Valpuesta, Shlosman \& Heller 2006; Chiba, Friske \& Schönrich 2021). In addition, while bars are generally vertically thin upon forming, they often drive a thickening of the inner disc which leads to the eventual formation of a boxy/peanut bulge (Combes \& Sanders 1981; Combes et al. 1990; Raha et al. 1991; Merritt $\&$ Sellwood 1994; Debattista et al. 2004; Martinez-Valpuesta et al. 2006). Bars are also found to trigger gas inflows which can lead to the formation of nuclear discs and resonant rings (e.g. Combes \& Gerin 1985; Buta \& Combes 1996; Sakamoto et al. 1999; Sheth et al. 2005; Cole et al. 2014). It is also possible for these gas inflows to fuel an active galactic nucleus, although at present there is no consensus on the efficacy of this process (see Combes 2003, Ho 2008, and references therein). The potential weakening and eventual destruction of bars has also been linked to the build-up of mass in the central regions (Hasan \& Norman 1990; Pfenniger \& Norman 1990; Friedli \& Benz 1993) or to the transfer of angular momentum from the infalling gas to the bar (Bournaud, Combes \& Semelin 2005), although high-resolution simulations have also found the opposite result (Shen \& Sellwood 2004; Athanassoula, Dehnen \& Lambert 2005; Debattista et al. 2006). In addition, bars are also capable of influencing the overall structure of the disc, for instance through the creation of a break in the radial density profile, which transforms a 
single-component disc into one with a double exponential radial density profile (Hohl 1971; Debattista et al. 2006; Minchev et al. 2012; Herpich et al. 2017). There have also been efforts to link the bar with the presence of counterrotating stellar components. Evans \& Collett (1994) found that stellar counterrotation may be induced by a dissolving bar, whereas Wozniak \& Pfenniger (1997) interpreted the 'wave pattern' found in the velocity curves of some barred S0 galaxies, within the region of the bar (Bettoni 1989; Bettoni \& Galletta 1997; Zeilinger et al. 2001), and in simulations (Pfenniger 1984; Sparke \& Sellwood 1987), to be the result of stars trapped on $x_{4}$ (quasi-circular retrograde) orbits by a bar. Given that bars have such a wide range of consequences for disc galaxies, we are motivated to investigate the degree to which they are capable of producing retrograde disc stars, and the implications this might have for disc galaxies such as the Milky Way (MW).

Dense, star-forming clumps in a young disc can also produce retrograde stars (e.g. Amarante et al. 2020b). High-redshift $(z>$ 1) galaxies often display a light distribution dominated by gas-rich, star-forming clumps, giving them an irregular, clumpy appearance. This contrasts with the smoother light distribution usually observed in massive disc galaxies in the local Universe. Clumps were first observed in high-redshift galaxies by Cowie, Hu \& Songaila (1995) and van den Bergh et al. (1996). The clumpy nature of early galaxies was confirmed by more recent observations, owing primarily to instruments capable of deep, high-resolution observations, such as Wide Field Camera 3 (WFC3) and the Near Infrared Camera and Multi-Object Spectrometer (NICMOS) on board the Hubble Space Telescope, which probe the rest-frame UV and near-infrared regions at $\mathrm{z}>1$ (e.g. Ravindranath et al. 2006; Elmegreen et al. 2007; Elmegreen 2009; Overzier et al. 2010; Swinbank et al. 2010; Förster Schreiber et al. 2011; Genzel et al. 2011; Guo et al. 2012). Their formation is likely due to gravitational instabilities in the proto-disc, a scenario which is supported by numerical studies (Noguchi 1999; Ceverino, Dekel \& Bournaud 2010). Guo et al. (2015) investigated the clump demographics of 3239 galaxies at redshift $0.5<z<$ 3.5 in the CANDELS/GOODS-S and UDS fields and found that $\approx 55$ per cent of intermediate-to-high-mass galaxies in their sample contain clumps. However, this fraction decreases at lower redshifts to $\approx 15$ per cent at $z=0.5$. On the other hand, $\approx 60$ per cent of lower mass galaxies have clumps, with this fraction remaining roughly constant with redshift. The sizes and masses of clumps are still somewhat uncertain, with $\sim 1 \mathrm{kpc}$ resolution observations yielding clump masses up to $10^{9.5} \mathrm{M}_{\odot}$ (Förster Schreiber et al. 2011; Soto et al. 2017). However, higher resolution studies have demonstrated that the derived masses and linear sizes of clumps are highly dependent on the resolution and sensitivity of observations, and are often systematically overestimated, with masses likely being closer to $\sim 10^{7}-10^{8} \mathrm{M}_{\odot}$ and linear sizes between 100 and $500 \mathrm{pc}$ (Dessauges-Zavadsky et al. 2017; Cava et al. 2018).

Significant work has also gone into understanding the impact that clumps have on the overall evolution of disc galaxies. For example, some studies find that if individual clumps are not disrupted by star formation and live long enough, they sink into the centre of the galaxy, forming a bulge component (Elmegreen et al. 2007; Dekel, Sari \& Ceverino 2009). More recently, clumps have also been proposed to be the driving mechanism behind the origin of the geometric and chemical thick discs (Bournaud, Elmegreen \& Martig 2009; Clarke et al. 2019; Beraldo e Silva et al. 2020b). In addition, using an isolated $\mathrm{N}$-body $+\mathrm{SPH}$ model similar to the MW, Amarante et al. (2020b) demonstrated that low angular momentum $\left(v_{\phi}<100 \mathrm{~km} \mathrm{~s}^{-1}\right)$ stars, including retrograde ones, are present in the Solar neighbourhood due to clump scattering in the first Gyrs
Table 1. The naming convention used reflects the absence/presence of clumps and bars. Whether or not the bar is long lived or decays is also reflected in the name.

\begin{tabular}{lc}
\hline Simulation name & Details \\
\hline M1_c_b & Clumps and bar \\
M2_c_nb & Clumps and no bar \\
M3_nc_b & No clumps and bar \\
M4_nc_bd & No clumps and decaying bar \\
\hline
\end{tabular}

of evolution, rather than exclusively because of the action of merger events, such as the Gaia-Enceladus-Sausage (Belokurov et al. 2018; Helmi et al. 2018), as previously suggested. The effect which clumps have on the evolution of galaxies such as the MW is not settled, but it remains possible that they may have played a significant role. Therefore, we also explore the role clumps play in driving retrograde motion in MW-like disc galaxies.

In this paper, we make a distinction between retrograde and counterrotating motion: while both retrograde and counterrotating stellar populations have the common characteristic of orbiting the host galaxy in an opposite sense to the main stellar disc, we emphasize that the retrograde populations we will explore do not take the form of a rapid counterrotating disc such as those observed in NGC 7217 (Merrifield \& Kuijken 1994) and NGC 4550 (Rix et al. 1992; Rubin, Graham \& Kenney 1992). Counterrotating discs occur when a substantial population of stars orbit the galaxy with a total angular momentum vector pointed in the opposite direction to that of the main stellar disc, and close to the circular velocity of the system. Counterrotation is generally thought to be linked with the accretion of counterrotating gas which subsequently forms stars (e.g. Katkov, Sil'chenko \& Afanasiev 2013; Pizzella et al. 2018). Given the right conditions, a counterrotating disc may also be produced by mergers (e.g. Puerari \& Pfenniger 2001). On the other hand, the retrograde populations we study in this paper do not rotate as rapidly, and indeed may or may not manifest as a disc, but may be more spheroidally distributed. Merger events have also been shown to heat orbits in a pre-existing disc and produce retrograde populations (e.g. Toth \& Ostriker 1992; Velazquez \& White 1999; Kazantzidis et al. 2009; Moetazedian \& Just 2016; Belokurov et al. 2020; Grand et al. 2020). However, the possibility of internal mechanisms such as the bar or stellar clumps being responsible for driving retrograde motion has not yet been fully explored.

This paper is organized as follows. Section 2 presents the simulations used in our analysis. In Section 3, we compare the models and show the properties of the retrograde populations in them. In Section 4, we discuss the implications of our results for the MW, and for disc galaxies in general. Section 5 presents a summary of our conclusions.

\section{THE SIMULATIONS}

We consider four $\mathrm{N}$-body + smooth particle hydrodynamics (SPH) simulations (see Table 1), spanning a range of evolutionary behaviours which provide us with a mixture of physical mechanisms to study. The initial conditions of all the models embed a gas corona in pressure equilibrium within a co-spatial Navarro-Frenk-White (NFW) (Navarro, Frenk \& White 1997) dark matter halo which constitutes 90 per cent of the mass. All stars form from the gas, with none present in the initial conditions. The first two simulations are M1_c_b and M2_c_nb. Both of these models formed clumps (hence the ' $c$ ' in the name), albeit with very different properties. Model 
M1_c_b formed a large-scale bar (hence the ' $b$ ' in the name), whereas M2_c_nb did not ('nb'). These two models have been presented in Ghosh, Debattista \& Khachaturyants (2020) and Beraldo e Silva et al. (2020a), respectively. Additional properties of these models can be found in those papers. Both models start with identical initial conditions but they are evolved with different subgrid physics. The initial conditions are a higher mass resolution version of the model described in Roškar et al. (2008).

The other two models both form bars; since they start from the same initial conditions, their evolution is very similar at first, until they diverge at later times because they implement different subgrid chemohydrodynamics evolutionary models. In the first one, M3_nc_b, the bar is long-lived ('b') while in M4_nc_bd the bar that forms subsequently decays ('bd'). Neither of these two models forms clumps ('nc'). Model M3_nc_b has been presented numerous times in previous papers; it is the star-forming model described most extensively in Cole et al. (2014) and Debattista et al. (2017). Model M4_nc_bd was described in Portaluri et al. (2017). The main difference is that in M4_nc_bd, thermal energy and metals can diffuse between gas particles, whereas this is prevented in model M3_nc_b. The evolution of the two models is initially quite similar, but their subsequent stochastic evolution sees them diverge when the gas inflow in M4_nc_bd overwhelms the bar, leading to its destruction. By the end of the simulations, a bar of radius $\approx 3 \mathrm{kpc}$ is present in M3_nc_b, while no bar is present in M4_nc_bd. We refer readers to Debattista et al. (2017) and Portaluri et al. (2017) for details of these two simulations.

\subsection{Simulation details for M1_c_b and M2_c_nb}

The initial conditions of these two models are identical. Their dark matter halo has a virial radius $r_{200} \simeq 200 \mathrm{kpc}$ and a virial mass $M_{200}=10^{12} \mathrm{M}_{\odot}$. The gas corona follows the same radial profile but constitutes only 10 per cent of the mass. No other baryons (and therefore no stars) are present at $t=0$. Gas particles are given a tangential velocity with cylindrical rotation such that the spin parameter $\lambda=$ 0.065 . Here, the spin parameter is defined as $\lambda \equiv J|E|^{1 / 2} /\left(G M_{\mathrm{vir}}^{5 / 2}\right)$, where $J$ and $E$ are the total angular momentum and the energy of the gas particles, and $G$ is the gravitational constant (e.g. Peebles 1969). The gas corona and the dark matter halo are comprised of $5 \times 10^{6}$ particles each, with softening parameters of $\epsilon=50 \mathrm{pc}$ (gas) and $\epsilon=100 \mathrm{pc}$ (dark matter). Star particles forming from the cooling gas also have a softening parameter of $\epsilon=50 \mathrm{pc}$.

The two models are evolved using these initial conditions for 10 Gyr with GASOLINE (Wadsley, Stadel \& Quinn 2004; Wadsley, Keller \& Quinn 2017) using different subgrid models for the physics of gas cooling, star formation, and supernova feedback. Both M1_c_b and M2_c_nb undergo an episode of clump formation early in their evolution. In the case of M2_c_nb, the inclusion of metal-line cooling of Shen, Wadsley \& Stinson (2010) allows the gas to cool more efficiently, which, together with the lower feedback, results in a more vigorous clumpy episode than in model M1_c_b. As gas cools, it settles into a disc and once the gas density exceeds $0.1 \mathrm{~cm}^{-3}$ star formation commences from gas particles with temperature below $15000 \mathrm{~K}$ which are part of a converging flow. In both models, star formation efficiency is set to 5 per cent. We use the blast wave supernova feedback recipe of Stinson et al. (2006). Supernova feedback couples 40 per cent of the $10^{51}$ erg per supernova to the interstellar medium as thermal energy in M1_c_b, and only 10 per cent in M2_c_nb. Gas mixing uses turbulent diffusion as described by Shen, Wadsley \& Stinson (2010). We use a base time-step of $\Delta t=5 \mathrm{Myr}$ with timesteps refined such that $\delta t=\Delta t / 2^{n}<\eta \sqrt{\epsilon / a_{g}}$, where we set the refinement parameter $\eta=0.175$. We set the opening angle of the tree-code gravity calculation to $\theta=0.7$. Gas particle time-steps also satisfy the condition $\delta t_{\mathrm{gas}}=\eta_{\text {courant }} h /\left[(1+\alpha) c+\beta \mu_{\max }\right]$, where $\eta_{\text {courant }}=0.4, h$ is the SPH smoothing length set over the nearest 32 particles, $\alpha$ and $\beta$ are the linear and quadratic viscosity coefficients, and $\mu_{\max }$ is described in Wadsley et al. (2004). Note that model M2_c_nb is comparable to a higher mass resolution version of the clump forming simulation described in Clarke et al. (2019), Beraldo e Silva et al. (2020b), and Amarante et al. (2020b). The set-up and simulation details of model M3_nc_b and M4_nc_bd are described at length in Cole et al. (2014), Debattista et al. (2017), and Portaluri et al. (2017); therefore, we do not repeat those details here.

A visual comparison of all four models can be made in Fig. 1, which shows synthetic RGB images of the stellar disc produced using PYNBODY (Pontzen et al. 2013) for each model at different points in time. In the first row, which represents the face-on stellar disc of each model after $1 \mathrm{Gyr}$, the clumpy nature of M1_c_b and M2_c_nb can be seen clearly, as opposed to M3_nc_b and M4_nc_bd, which do not undergo a clumpy episode. Models M1_c_b, M3_nc_b, and M4_nc_bd all undergo bar formation, but only M1_c_b and M3_nc_b manage to keep their bars. While M3_nc_b never undergoes bar destruction, model M4_nc_bd (right column) starts to undergo bar dissolution at $\approx 4 \mathrm{Gyr}$. Indeed, while a remnant of the bar is still present at $5 \mathrm{Gyr}$ (third row), this is all but gone by the end of the simulation at $10 \mathrm{Gyr}$ (fifth row). The even-numbered rows in Fig. 1 show the evolution of the simulations represented in the side-on view. The clumps can be seen occupying the mid-plane in models M1_c_b and M2_c_nb at 1 Gyr. Finally, these two have a thicker disc compared to M3_nc_b and M4_nc_bd throughout their evolution due to the scattering generated by the clumps.

Ideally, we would also compare results from the models against a control model which does not have either a bar or clumps to estimate the contribution of retrograde motion due to numerical heating. However, to some extent all simulations form at least very weak bars. Despite this, as will be shown in Sections 3.2 and 3.3, our results indicate that, in the absence of bars and clumps, the fraction of retrograde stars remains constant. Additionally, we find no evidence that retrograde motion is driven by radial heating due to spirals in the models. We conclude that the retrograde fraction driven by numerical noise must be relatively small.

\section{RESULTS}

We now compare the models and investigate the properties of the retrograde populations in them. In Section 3.1, we quantify the evolution of clumpiness and bar strength in the models. In Section 3.2, we investigate to what extent both perturbations produce retrograde orbits. In Section 3.3, we investigate the spatial extent to which bars and clumps drive retrograde motion. Finally, in Section 3.4, we analyse the age distributions and some of the orbital characteristics of the retrograde stars.

\subsection{The clumpiness and bar strength of the models}

In order to assess the relative efficiencies of bars and clumps at producing retrograde stars, we first need to quantify the strengths of both perturbations. Models M1_c_b and M2_c_nb undergo an early episode of clump formation. To measure the strength and duration of the clumpy episodes, we start by constructing 2D-histograms of the density of the models at each time-step. Each histogram measures $N_{x} \times N_{y}=400 \times 400$ bins, corresponding to a region $20 \times 20 \mathrm{kpc}$ 

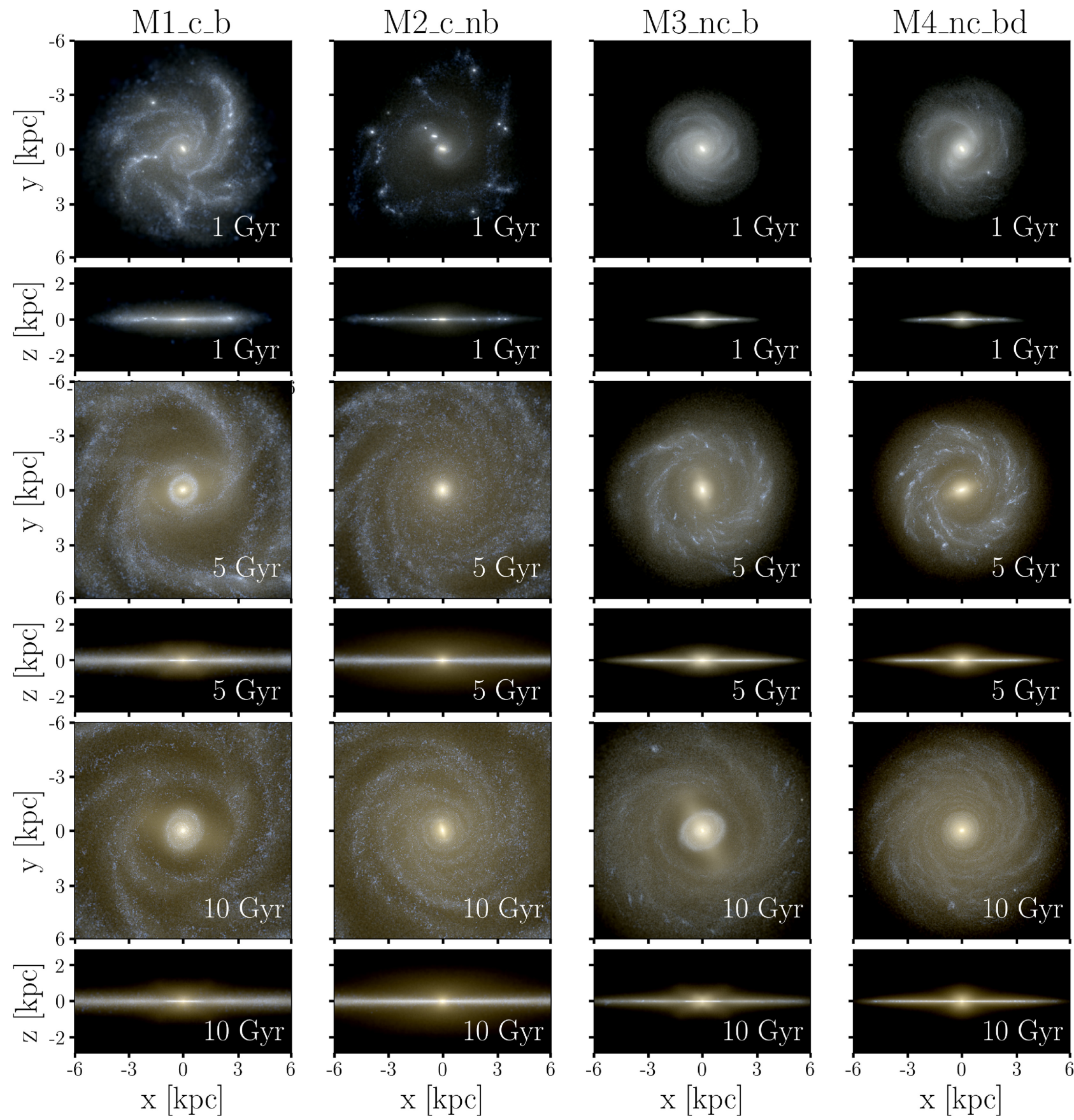

Figure 1. Synthetic RGB images produced with PYNBODY of the stellar discs of the models. We show the models both face-on and side-on at 1 Gyr (top two rows), then at $5 \mathrm{Gyr}$ (next two rows), and at $10 \mathrm{Gyr}$ (bottom two rows). These images highlight the different evolutionary paths the models take. The clumpy nature of M1_c_b and M2_c_nb early in their evolution may also be contrasted with M3_nc_b and M4_nc_bd, which do not go through a clumpy episode.

in size, and represents the mass distribution of the models seen faceon. We identify the clumps as overdensities in the mass distribution by employing the FIND_PEAKS function in the PHOTUTILS Python package (Bradley et al. 2020). This detects any local maxima in the mass distribution which are above a (manually set) density threshold, given by $\zeta=M+(150 \sigma)$, where $M$ and $\sigma$ are the median and standard deviation of the mass distribution. Having located the peaks, on the face-on view of the models we construct a circle of radius $150 \mathrm{pc}$ around each clump to isolate the stars constituting it. While the clumps in the models vary in size, we found that a radius of $150 \mathrm{pc}$ is sufficient to capture the mass contained within them. Moreover, this yielded an average clump mass of $\sim 10^{8} \mathrm{M}_{\odot}$ for both of the clumpy models, which is in agreement with high-resolution observations (e.g. Cava et al. 2018). The threshold was optimized to ensure that clump detection minimized contamination from other sources, such as spiral arms. We then determine the total stellar mass contained in all the clumps, and normalize it by the total stellar mass of the model during each time-step; this gives us the clump mass fraction, $\chi_{\text {clumps }}$, as a function of time. While this method of detecting clumps also picked up the central mass concentrations (bulges) in the models, these were not included in the calculations of the clumpy mass fraction. 


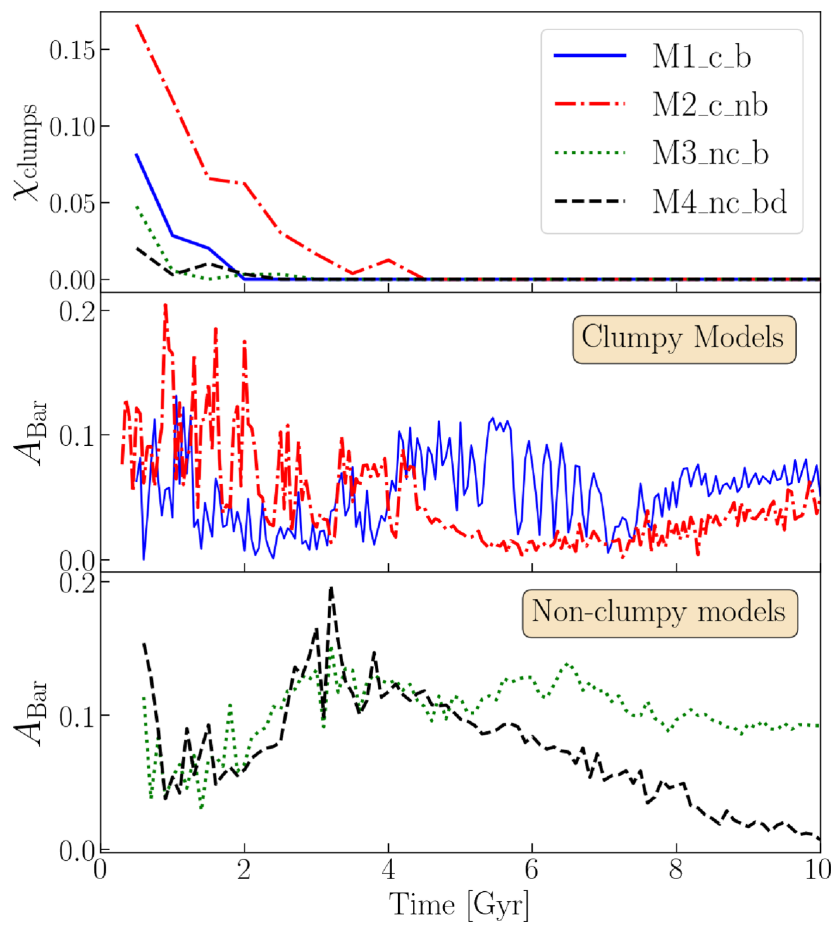

Figure 2. Evolution of perturbations in the models. Top: the clumpy mass fraction $\chi_{\text {clumps }}$. While M1_c_b and M2_c_nb both undergo a clumpy episode early in their evolution, M2_c_nb has stronger clumping which lasts until about 4.5 Gyr. On the other hand, the clumpy episode in M1_c_b is over by 2.5 Gyr. Models M3_nc_b and M4_nc_bd do not suffer any significant clumping. Middle: evolution of the bar strength, given by the $m=2$ Fourier moment, for the clumpy models. While bar formation in M1_c_b starts at $\approx 4$ Gyr and results in a long-lived bar, model M2_c_nb does not develop a large-scale bar. The non-zero value for $A_{\mathrm{bar}}$ for this model results from the clumps, which show up as $m=2$ perturbations. Bottom: evolution of the bar strength for the non-clumpy models, M3_nc_b and M4_nc_bd. Both models undergo early bar formation, which peaks at $\approx 4$ Gyr. However, while the bar in M3_nc_b is long-lived, the bar in M4_nc_bd starts to decay after reaching its peak amplitude, dissolving entirely by the end of simulation.

The top panel of Fig. 2 shows the evolution of $\chi_{\text {clumps }}$ for the four models. Models M1_c_b and M2_c_nb both undergo an early clumpy episode. These clumps can also be seen in the top row of Fig. 1. Overall, model M2_c_nb is more clumpy than model M1_c_b, and the clumpy episode lasts longer, until 4.5 Gyr (compare with Clarke et al. 2019), after which no further clumps form. Model M1_c_b suffers a milder episode of clump formation, which is over by $2.5 \mathrm{Gyr}$. Models M3_nc_b and M4_nc_bd show no significant clumping throughout their evolution. The non-zero value for $\chi_{\text {clump }}$ for these models in the first 2 Gyr can be attributed largely to spiral contamination, which could not be completely avoided in these models.

The bar strength is measured in the usual way as the amplitude of the global $m=2$ Fourier moment, $A_{\mathrm{Bar}}$, of the face-on mass distribution (e.g. Sellwood \& Athanassoula 1986; Debattista et al. 2006). The middle and bottom panels of Fig. 2 show the evolution of $A_{\mathrm{Bar}}$. Bar formation in M1_c_b (middle panel, solid blue line) starts at $\sim 4 \mathrm{Gyr}$ and results in a long-lived bar (see left column of Fig. 1). Model M2_c_nb (middle panel, dashed red line), on the other hand, does not form a large-scale bar throughout its evolution, as can be seen in Fig. 1. The non-zero value for $A_{\mathrm{Bar}}$ for this model is a result of its clumpy nature in the first few Gyr. Indeed, this signal may be attributed to clumps which happen to be on opposite sides of the disc being picked up as $m=2$ perturbations, resulting in a fake signal. In addition, the bar amplitude for M2_c_nb also drops as soon as the clumpy episode in M2_c_nb ends at $4.5 \mathrm{Gyr}$. Spiral arms are also present in models M1_c_b and M2_c_nb (see Fig. 1), which interfere with the $A_{\mathrm{Bar}}$ signal, generating the fluctuations in the data.

In models M3_nc_b and M4_nc_bd (bottom panel of Fig. 2), bar formation starts at $\sim 2 \mathrm{Gyr}$, and reaches peak strength at $\sim 4$ Gyr. Up until this point in time, the evolution of the bar in both models is very similar. However, the bar in model M3_nc_b is long-lived, whereas the bar in M4_nc_bd slowly starts to weaken at 4 Gyr, eventually dissolving entirely.

\subsection{Bars and clumps as drivers of retrograde motion}

In this subsection, we show that bars and stellar clumps play a role in driving retrograde motion of disc stars in the models. Fig. 3 shows the time evolution of the total mass fraction of retrograde stars, $f_{\text {ret }}=M_{\text {ret }}(t) / M_{\star}(10 \mathrm{Gyr})$ in the models, where $M_{\text {ret }}(t)$ is the total mass of retrograde stars at time $t$, and $M_{\star}(10 \mathrm{Gyr})$ is the total stellar mass at the end of the model's evolution (10 Gyr), respectively. While the red lines track $f_{\text {ret }}$ for the entire models, the blue lines represent the retrograde mass fraction inside a cylindrical galactocentric radius of $5 \mathrm{kpc}$, and are normalized by the total stellar mass in this region. The solid and dashed grey lines reflect the clumpy mass fraction and the bar amplitude, respectively.

Model M2_c_nb (top right panel in Fig. 3), which forms clumps but no large-scale bar, has a population of retrograde stars which increases continuously up until $4.5 \mathrm{Gyr}$ (red line), when it reaches a plateau. This coincides with the point at which the last clumps in this model dissolve. When considering the entire model (red line), model M2_c_nb reaches $f_{\text {ret }} \simeq 0.06$ by the end of the clumpy epoch (4.5 Gyr). Retrograde stars at $4.5 \mathrm{Gyr}$ represent 10 per cent of the total stellar mass at that time. After the clumpy epoch, $f_{\text {ret }}$ remains roughly constant up until 8 Gyr. This indicates that, in the absence of both clumps and a bar, additional retrograde stars are not being produced in any significant amount via other forms of scattering, whether physical or numerical. The slight increase in the retrograde fraction after $8 \mathrm{Gyr}$ can be attributed to the formation of a small, $1 \mathrm{kpc}-\mathrm{scale}$, bar during this time, which can be seen in Fig. 1 (second column). Clumps also drive retrograde motion in M1_c_b (top left panel in Fig. 3). Due to its weaker clump formation episode (grey line), it only reaches $f_{\text {ret }} \simeq 0.03$ during its initial clumpy stage, which ends at roughly $2.5 \mathrm{Gyr}$. Retrograde stars at $2.5 \mathrm{Gyr}$ represent 6 per cent of the total stellar mass at that time. However, once the bar starts to form at around $4 \mathrm{Gyr}, f_{\text {ret }}$ roughly doubles during a short time interval ( $\sim 2 \mathrm{Gyr})$ before reaching a peak, and increases slowly thereafter.

Models M3_nc_b and M4_nc_bd (bottom row), which do not suffer any significant clumping but form bars early in their evolution, both develop a large retrograde population, with both models reaching $f_{\text {ret }} \simeq 0.14$ by $10 \mathrm{Gyr}$. In model M3_nc_b (bottom left panel), $f_{\text {ret }}$ increases continuously as the bar grows until roughly $7 \mathrm{Gyr}$, when the growth of $f_{\text {ret }}$ slows down, corresponding to the weakening of the bar at this time (see dashed grey line). In model M4_nc_bd (bottom right panel), $f_{\text {ret }}$ increases steadily as the bar grows until roughly 3 Gyr. However, as the bar dissolves (see dashed grey line) we see that while retrograde stars are still being produced, this happens at a continuously declining rate.

The solid blue lines, which reflect $f_{\text {ret }}$ inside $R=5 \mathrm{kpc}$, are normalized by the total stellar mass inside this region. In general, 

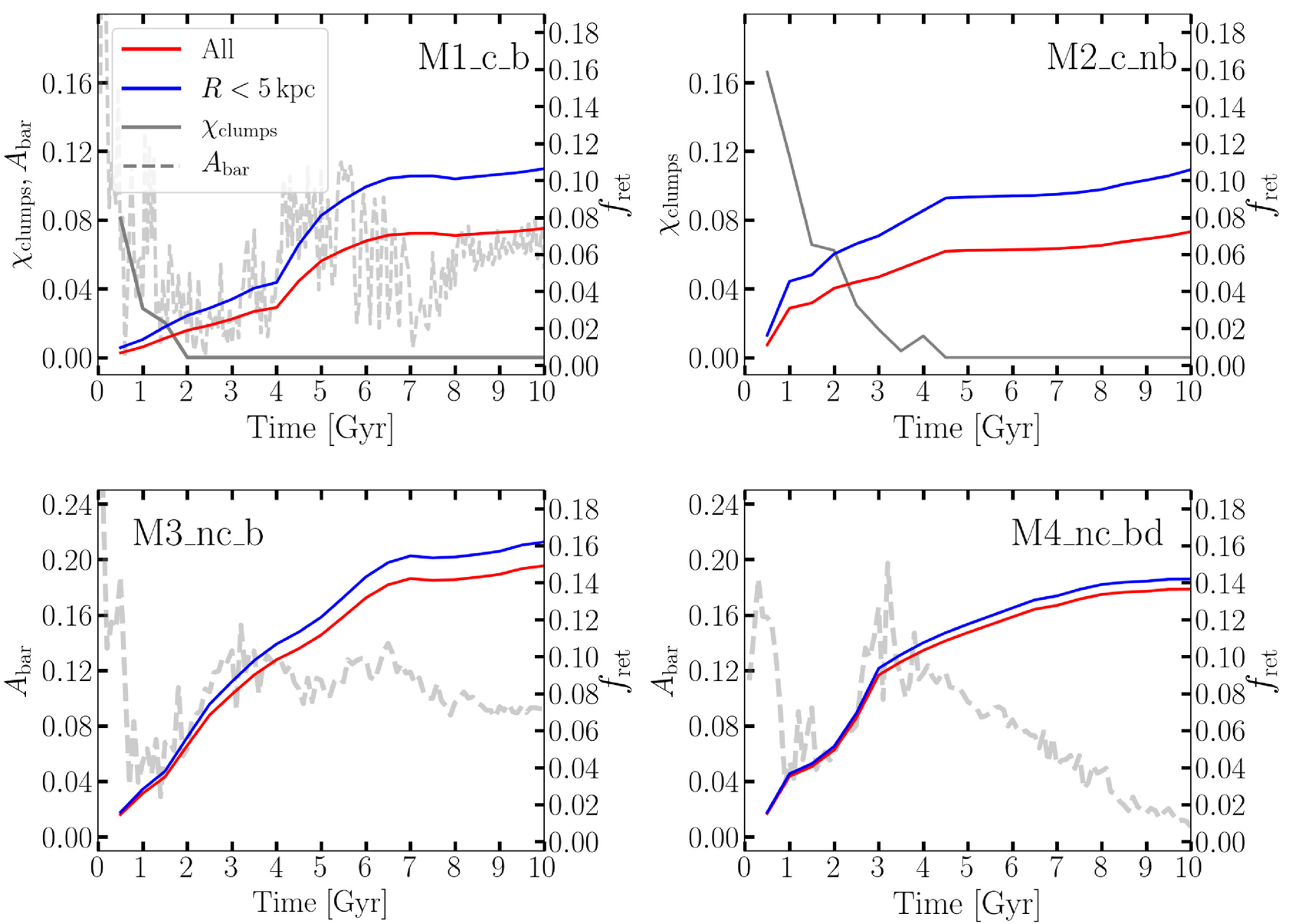

Figure 3. The time evolution of the retrograde mass fraction, $f_{\mathrm{ret}}=M_{\mathrm{ret}}(t) / M_{\star}(10 \mathrm{Gyr})$, in the four models, where $M_{\mathrm{ret}}(t)$ and $M_{\star}(10 \mathrm{Gyr})$ are the total mass of retrograde stars at time $t$ and the total stellar mass at the end of the models, respectively. The red lines show $f_{\text {ret }}$ for all stars, while the blue lines reflect $f_{\text {ret }}$ inside $5 \mathrm{kpc}$, with $M_{\star}(10 \mathrm{Gyr})$ being the total stellar mass also inside this region. The solid and dashed grey lines reflect the clumpy mass fraction and the bar amplitude, respectively. In both cases the scales for the grey lines are presented on the left hand axis.

we find that $f_{\text {ret }}$ is higher inside $R=5 \mathrm{kpc}$, indicating that these retrograde populations are centrally concentrated.

Fig. 4 shows the distribution of $V_{\phi}$ versus cylindrical galactocentric radius, $R$, for all stars in the models at three different times. Both of the clumpy models, M1_c_b and M2_c_nb, have peaks at $V_{\phi}$ $\approx 0$, even during the earliest time at $1 \mathrm{Gyr}$ (first row). This is due to the clumps having already scattered stars by this time. Additionally, while the $V_{\phi} \approx 0$ peak in model M1_c_b seems to be distinct from the main stellar population, especially at later times, this is not the case in model M2_c_nb, which shows a more continuous transition. This is likely due to the stronger clumpy episode in M2_c_nb, which has driven larger amounts of scattering, and as a result produces a continuous distribution. The vertical overdensities in models M1_c_b and M2_c_nb at $1 \mathrm{Gyr}$ are due to the presence of the clumps. On the other hand, the non-clumpy models M3_nc_b and M4_nc_bd are not peaked at $V_{\phi} \approx 0$, with the retrograde populations being continuous with the main stellar population.

We conclude that both bars and clumps can produce a retrograde population of order 10 per cent (when considering the whole galaxy). This is a fairly significant population. We find that bars are slightly more efficient at producing retrograde stars, but the difference is not very large.

\subsection{The spatial distribution of retrograde stars}

Having established that both clumps and bars drive stars to retrograde motion, we shift our attention to the spatial distribution of these retrograde stars. Fig. 5 shows the radial density profiles, $\left(\Sigma_{*}\right)$, for the models at different times. The left-hand column represents the density profiles for all stars in the models, while the middle column shows the radial profiles of just the retrograde stars. The left-hand column shows the usual inside-out formation of discs (e.g. Chiappini, Matteucci \& Romano 2001; Muñoz-Mateos et al. 2007; Bovy et al. 2012; Frankel et al. 2019), with the density profile extending to increasingly large radii with the passage of time. This is in part due to the increasingly large gas disc, but is also the result of angular momentum exchange, which leads to the migration of stars to ever larger radii (e.g. Roškar et al. 2008; Sharma, Hayden \& BlandHawthorn 2020).

The middle column of Fig. 5 shows the differences amongst the four models in the distribution and evolution of the retrograde stars. First, in all cases, the radial density profile of retrograde stars shows only marginal evolution as time progresses when compared to the rest of the stellar populations. At $R \gtrsim 5 \mathrm{kpc}$, the density of retrograde stars at $2 \mathrm{Gyr}$ (green line) is higher than at all other times; this reflects on an ongoing spray of stars that has not yet settled into a stationary distribution. 

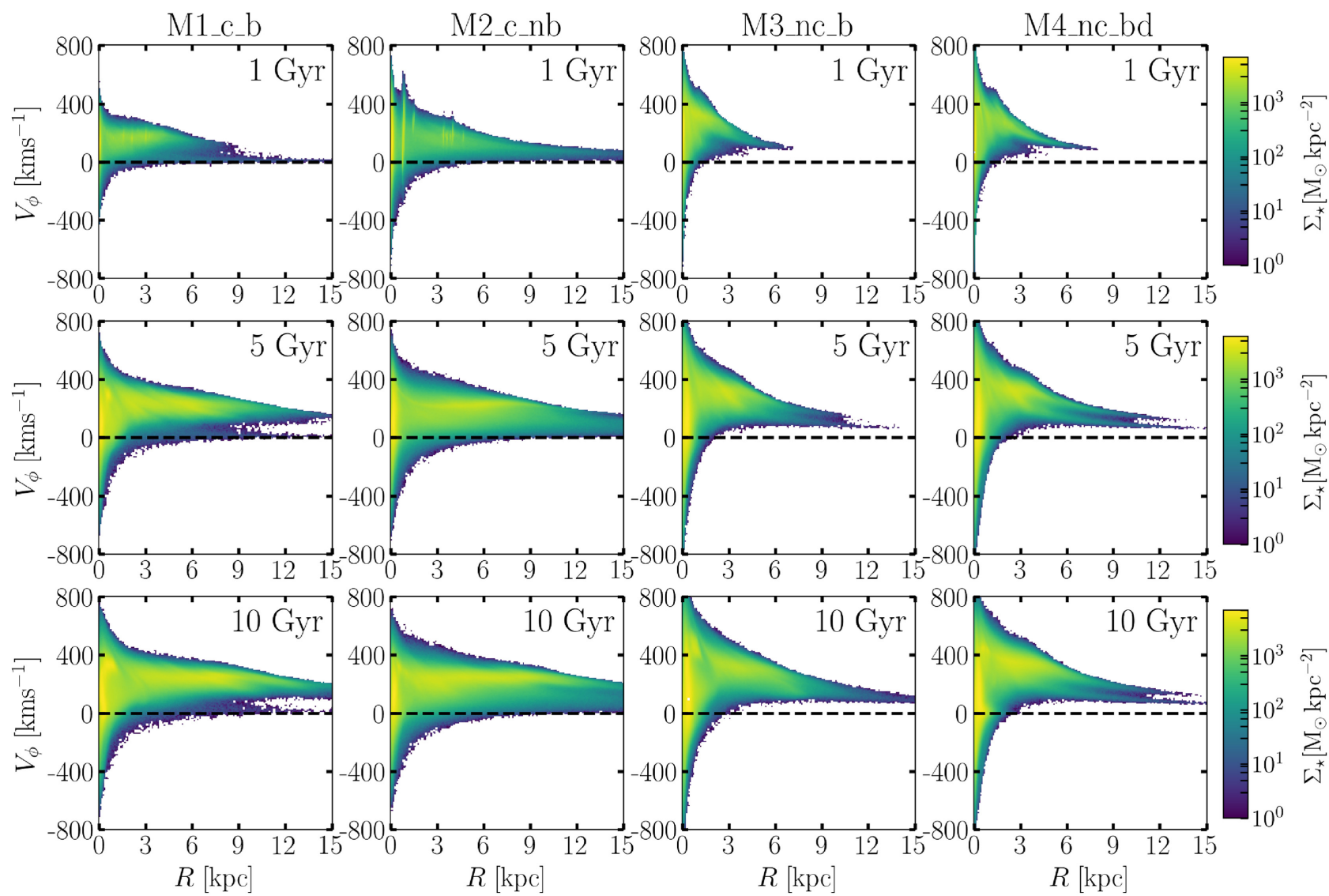

Figure 4. Density distributions in the $V_{\phi}$ versus cylindrical galactocentric radius, $R$, plane, of stars in the models at three different times. The retrograde population in the clumpy model M1_c_b (first column) shows itself as a distinct peak centred around $V_{\phi} \approx 0$ and is present even at 1 Gyr due to clumps already having scattered stars by this time. This is also the case in model M2_c_nb, which has stronger clumps. However, the peak centred at $V_{\phi} \approx 0$ is continuous with the main stellar population. The vertical overdensities in M1_c_b and M2_c_nb at $1 \mathrm{Gyr}$ are due to the presence of clumps at that time. The non-clumpy models, M3_nc_b and M4_nc_bd, lack a peak at $V_{\phi} \approx 0$, with the retrograde populations being present only in the central regions.

The radial extent of retrograde stars is very different between clumpy and non-clumpy models. This can be seen in the right column of Fig. 5, where we show the mass fraction of retrograde stars, $f_{\text {ret }}$, in each radial bin, at $10 \mathrm{Gyr}$ for each model. In models M3_nc_b and M4_nc_bd, neither of which formed clumps, the retrograde population remains confined within the immediate vicinity of the bar. We also find that bar-driven retrograde stars remain in the region of the bar even after the bar dissolves. On the other hand, the profiles for models M1_c_b and M2_c_nb are more radially extended. The retrograde population is most radially extended in model M2_c_nb, which had the most vigorous clump-formation episode. However, even the weaker clumpy episode of M1_c_b still produces an extended profile, showing that even mild clump formation can cause retrograde stars to be scattered to large radii.

This contrast in the radial extent of bar-driven and clump-driven retrograde stars can also be seen in Fig. 6, where we plot the density distribution of these stars in the $(x, z)$ Cartesian plane, for different bins of angular momentum, $L_{\mathrm{z}}$. We see again that the retrograde stars in the clumpy models (first two columns in Fig. 6) reach larger galactocentric radii than those in the non-clumpy models (second two columns), for all bins of $L_{z}$ (i.e. in all rows). Clump-driven retrograde stars also reach larger heights above the mid-plane, as scattering by clumps converts in-plane motions to vertical ones. In addition, in the bin with the most negative angular momentum (bottom row), the retrograde stars form a relatively flattened distri- bution, becoming more spherical as $L_{z}$ increases (middle and top rows).

The lack of retrograde stars in the outer regions $(R>5 \mathrm{kpc})$ of the non-clumpy models rules out the possibility for retrograde motion being driven by radial heating due to spirals. If this were the case, then we would observe a population of retrograde stars in the outer discs of these models where the spirals are present. However, we find no evidence for this.

We conclude that while bars and clumps both drive retrograde motion in disc stars, bars are not efficient at producing retrograde stars at large radii. This tendency for bar-driven retrograde stars to remain in the vicinity of the bar might possibly be due to the presence of the bar resonances. Indeed, stars whose orbits are in the vicinity of the resonances of a growing bar perturbation may be captured into librating orbits (e.g. Kalnajs 1973; Tremaine \& Weinberg 1984; Collett, Dutta \& Evans 1997). Consequently, the bardriven retrograde stars will be unable to escape the bar. On the other hand, while a large proportion of the clump-driven retrograde stars also remains in the inner regions of the simulated galaxies, scattering by the clumps is more likely to drive these stars to larger radii.

\subsection{Ages and orbits of retrograde populations}

Fig. 7 shows the distribution of stellar ages versus the circularity parameter (at $10 \mathrm{Gyr}$ ), defined as $\lambda_{z} \equiv L_{z} / L_{c}(\mathrm{E})$, where $L_{c}(\mathrm{E})$ is 

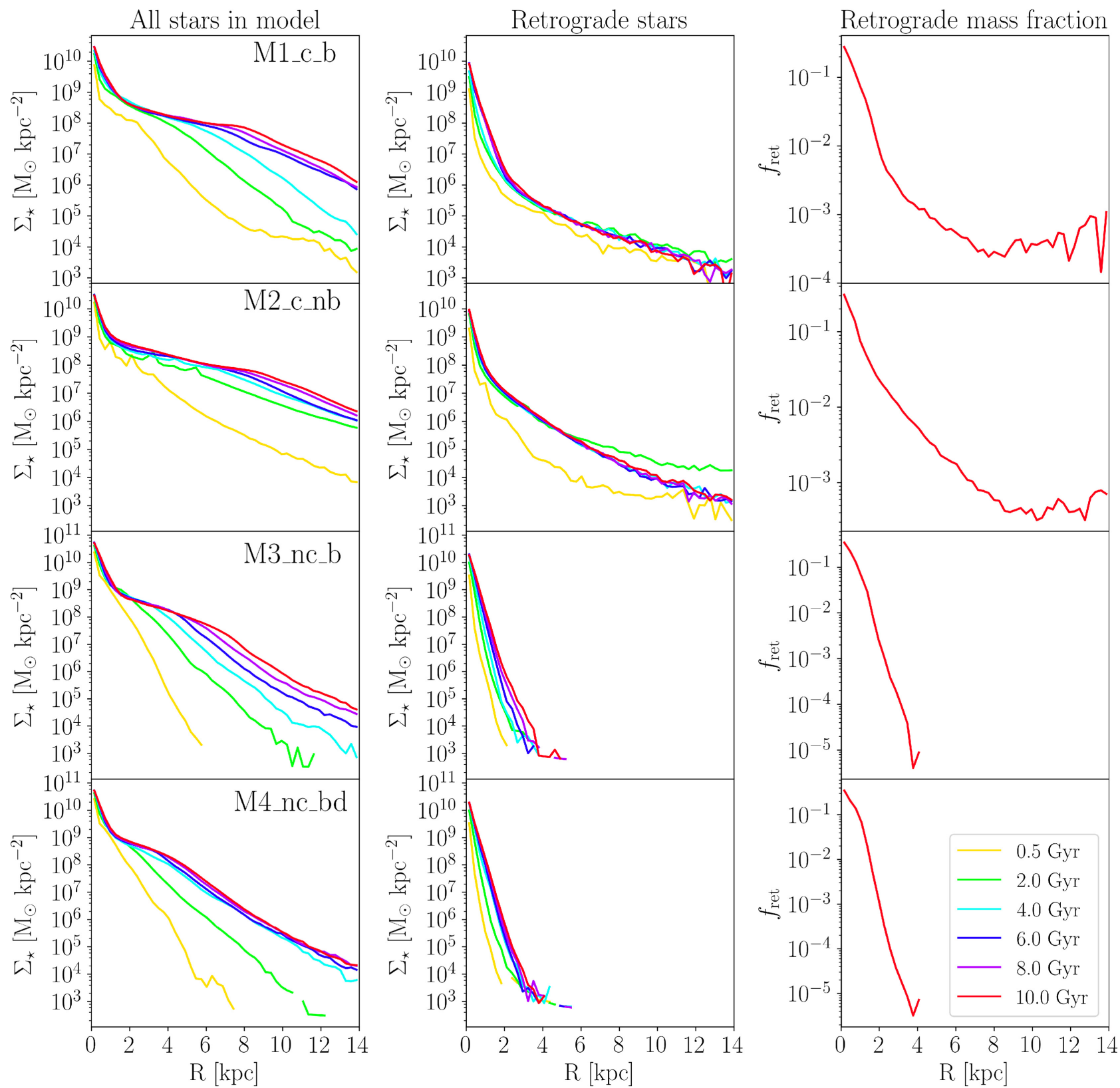

Figure 5. Left: the evolution of the radial density profiles for all stars in each model. Middle: the evolution of the radial density profiles for retrograde stars only. Right: the profile of the mass fraction of retrograde stars, $f_{\text {ret }}$, at $10 \mathrm{Gyr}$ for each model, which reflects the radial distribution of retrograde stars at the end of each model's evolution. The full profiles show substantial radial growth with time, while the profiles for the retrograde stars exhibit only minimal outward growth. The radial extent of the retrograde stars is strongly dependent on whether clumps were present in the early epochs or not. Even a mild episode of clump formation is sufficient to extend the retrograde stars to larger radii, whereas the retrograde stars driven by the bar tend to remain within the vicinity of the bar.

the angular momentum of a particle with binding energy $E$ to be on a circular orbit (Abadi et al. 2003). In computing $L_{c}(\mathrm{E})$, we assume an axisymmetric disc, and that the orbit lies in the midplane. Therefore, some caution must be exercised in interpreting the circularity distribution for stars in the region of the bar. The top row, which presents the distribution for stars in the outer regions $(R>5 \mathrm{kpc})$, shows that in models M1_c_b and M2_c_nb, the retrograde stars were all born during the clumpy epoch (indicated by the horizontal, shaded region). Fig. 5 showed that bars are unable to drive significant retrograde orbits much beyond their radius. This is also reflected in the upper panels for models M3_nc_b and M4_nc_bd (right columns), where virtually no retrograde stars inhabit this outer region. The bottom row of Fig. 7 shows that retrograde stars in the inner regions $(R<5 \mathrm{kpc})$ span a much wider range of ages. This is expected since a long-lived bar will continuously produce retrograde orbits throughout its life.

Fig. 7 also shows that most of the retrograde orbits produced by clumps in the outer regions have near-zero or low circularities. This is most apparent in the upper panel for models M1_c_b and M2_c_nb, where retrograde stars have circularity in the range $\lambda_{z}=$ $-0.5-0$. Retrograde stars in the inner regions (bottom row) also span a wider range of $\lambda_{z}$, with the most circular orbits (higher negative $\lambda_{z}$ ) being attained by the oldest stars. We further characterize the motion of the retrograde populations in Fig. 8, where we plot the 

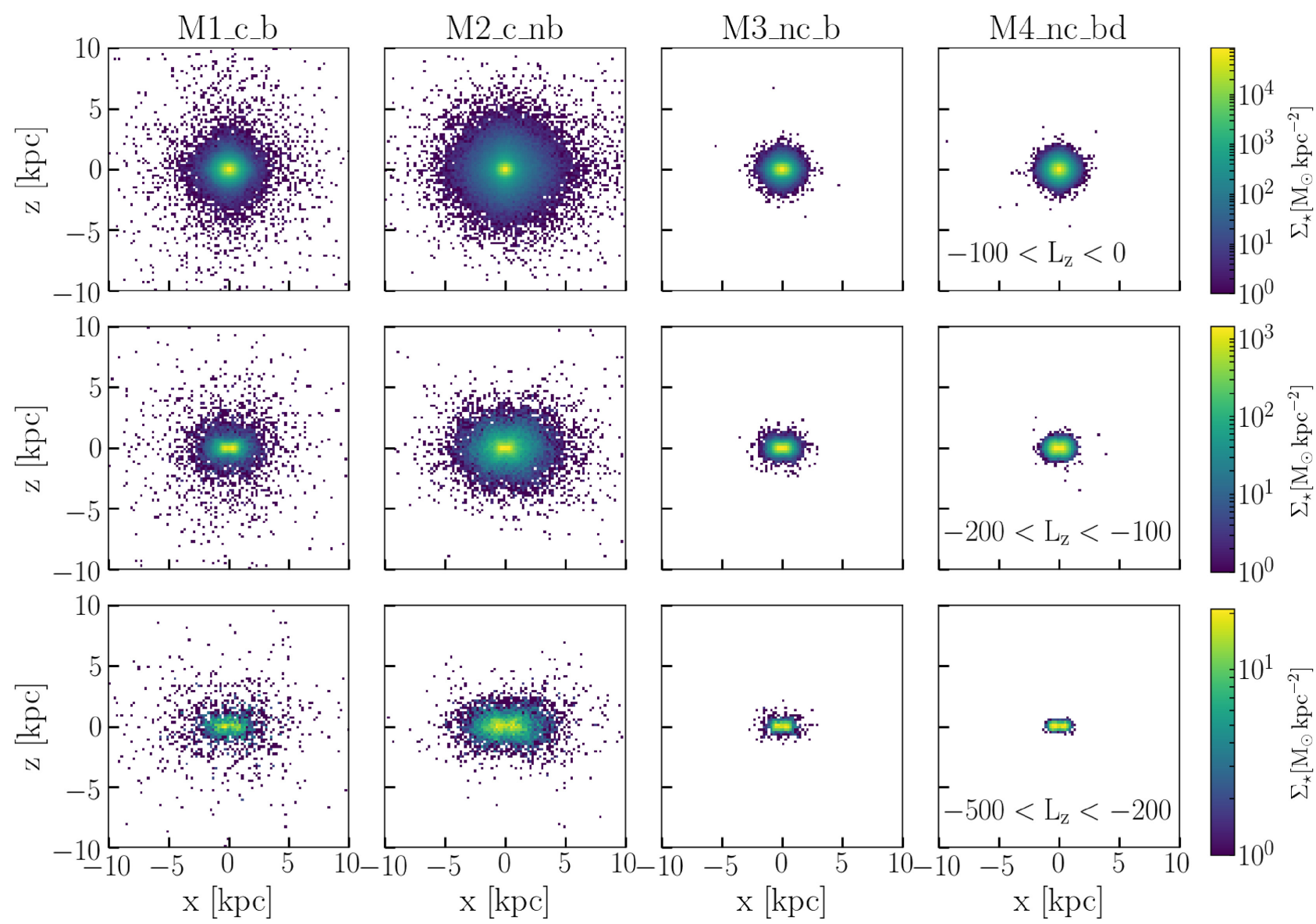

Figure 6. Density distribution plots in $(x, z)$ plane of retrograde stars at $10 \mathrm{Gyr}$ for each of the models. Each row represents the density distribution of retrograde stars in different angular momentum $\left(L_{z}\right)$ bins. The retrograde stars in M1_c_b and M2_c_nb (first two columns) are found at larger radii due to the clump scattering for all $L_{z}$ ranges, compared to centrally concentrated retrograde population in M3_nc_b and M4_nc_bd. In addition, it is evident that bars also cause some vertical heating, causing some of the bar-driven retrograde populations to be nearly spherically distributed.

distribution of retrograde stars in the space of $L_{\mathrm{z}} / L_{\mathrm{tot}}$ versus the spherical radius $r_{\text {sph }}$ for the models at $10 \mathrm{Gyr}$. In models M1_c_b and M2_c_nb (upper row), the outer regions $\left(r_{s p h}>5 \mathrm{kpc}\right)$ are clearly dominated by retrograde stars having low values of $L_{\mathrm{z}} / L_{\mathrm{tot}}$, which indicates that much of the angular momentum of these stars is not perpendicular to the disc plane. Combined with their low circularity, it suggests that clump-driven retrograde stars are predominantly on boxy orbits. On the other hand, orbits span a wider range of $L_{\mathrm{z}} / L_{\mathrm{tot}}$ in the inner region where the bar dominates. However, we have also seen in Fig. 6 that bars produce a retrograde population which has a spheroidal, rather than a discy, distribution.

\section{OBSERVATIONAL CONSEQUENCES}

We now consider the observational implications of our results for the MW, and for disc galaxies in general.

\subsection{Implications for the MW}

We have demonstrated that while bar-driven retrograde stars remain in the vicinity of the bar, clumps are capable of driving retrograde motion to larger galactocentric radii (Figs 5 and 6). Therefore, we expect that a population of clump-driven retrograde stars will be present in the Solar neighbourhood if the MW has experienced a clumpy episode. On the other hand, we do not expect any bar- driven retrograde stars to be present in this region, as these will be restricted to Galactocentric radii comparable to the MW's bar. In the clumpy models we found the retrograde mass fraction, $f_{\text {ret }}$, in the Solar cylinder (defined as $7.5<R_{\mathrm{G}} / \mathrm{kpc}<8.5$ and $0<|z| / \mathrm{kpc}$ $<2$ ) to be 0.01 per cent and 0.04 per cent for M1_c_b and M2_c_nb, respectively. The clump-driven retrograde stars in the models are part of the Splash-like population studied in Amarante et al. (2020b). They found the relative fraction of this population, defined as the metal-rich $(-0.7<[\mathrm{Fe} / \mathrm{H}]<-0.2)$, low angular momentum $\left(v_{\phi}<100 \mathrm{~km} \mathrm{~s}^{-1}\right)$ stars in an isolated clumpy simulation, ${ }^{1}$ is in good agreement with the fraction observed in the MW using the same selection criteria (see their fig. 4, left-hand panel).

We estimate the MW's Solar neighbourhood retrograde fraction (those within $1 \mathrm{kpc}$ of the Sun) based on the local fraction of the kinematically defined halo, which is $0.45-0.60$ per cent (Kordopatis et al. 2013; Posti et al. 2018; Amarante, Smith \& Boeche 2020a). Since this population's velocity distribution is well fit by a Gaussian, with mean $v_{\phi} \sim 20 \mathrm{~km} \mathrm{~s}^{-1}$ and $\sigma_{\phi}=85-100 \mathrm{~km} \mathrm{~s}^{-1}$, roughly 40 per cent of these stars are on retrograde orbits. The resulting fraction of retrograde stars in the Solar neighbourhood therefore is $\approx 0.18-0.24$ per cent. This fraction has no chemical selection; for this reason, its higher value compared to the models

\footnotetext{
${ }^{1}$ Amarante et al. (2020b) studied a lower mass resolution version of M2_c_nb.
} 

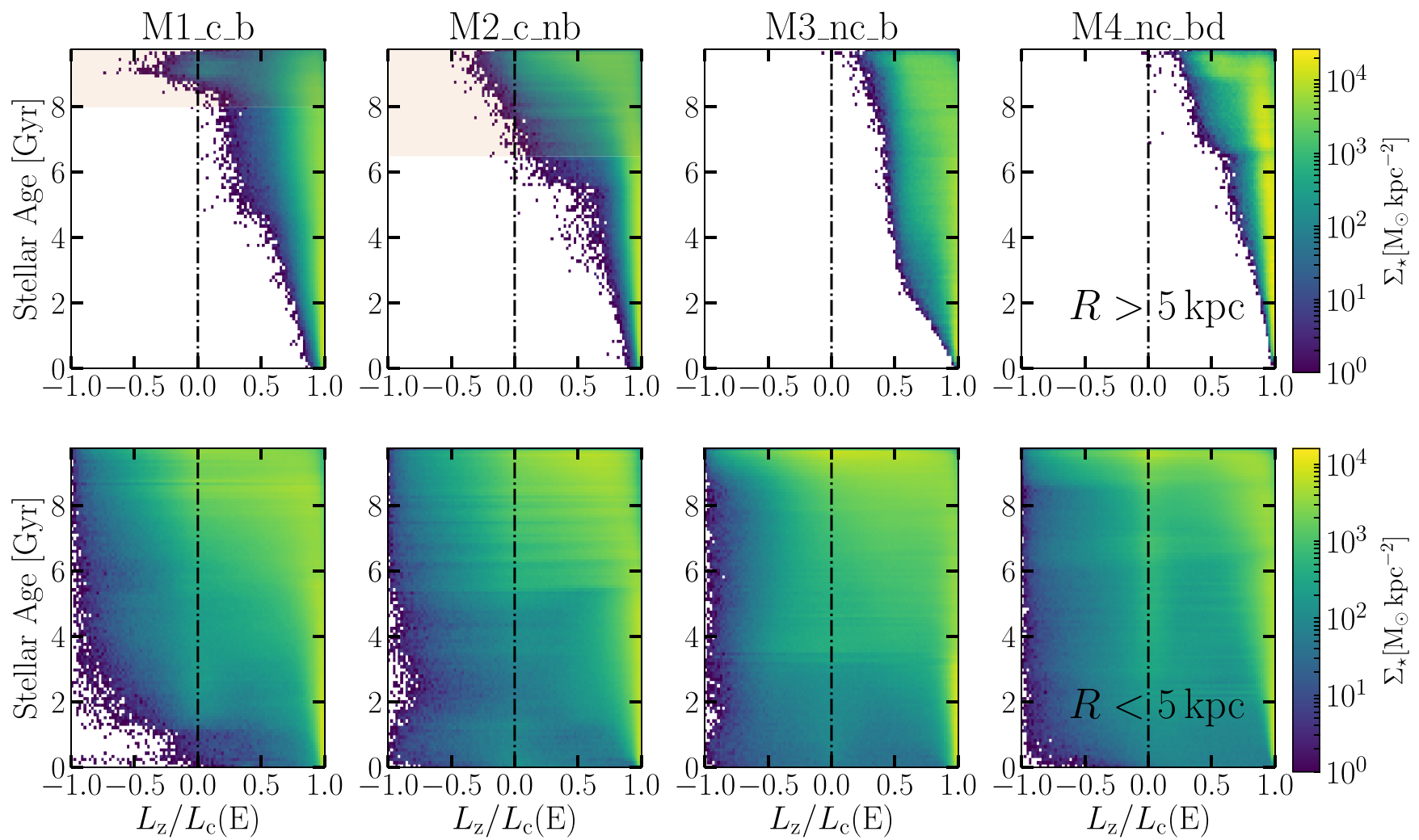

Figure 7. Distribution of stellar age versus orbital circularity, $\lambda_{z}=L_{z} / L_{\mathrm{c}}(\mathrm{E})$, for stars at $R>5 \mathrm{kpc}$ (top row) and $R<5 \mathrm{kpc}$ (bottom row) at $10 \mathrm{Gyr}$. Stars in the shaded regions in the upper row are born during the clumpy episodes of M1_c_b and M2_c_nb. In the outer region, the only retrograde stars present are those which were born during the clumpy epoch. Models M3_nc_b and M4_nc_bd have no retrograde stars in this outer region. In the inner regions (bottom row), retrograde stars span a much wider range of ages. This is due to the heating of stars by the bar throughout its lifetime. In the case of M2_c_nb, the presence of younger retrograde stars in the inner region is likely due to a small bar which forms at roughly $8 \mathrm{Gyr}$.

is attributed to the fact that it also includes halo stars accreted by the MW (e.g. Hawkins et al. 2015; Belokurov et al. 2018; Hayes et al. 2018; Helmi et al. 2018; Das, Hawkins \& Jofré 2020). The models only have in situ retrograde stars, and their lower fraction shows that retrograde orbits are not overproduced due to excessive scattering in the models. Moreover, a considerable number of retrograde stars associated with the Gaia-Enceladus-Sausage event (Belokurov et al. 2018; Helmi et al. 2018) lie exactly in the region associated with the thick disc in the $[\mathrm{Mg} / \mathrm{Fe}]-[\mathrm{Fe} / \mathrm{H}]$ plane (see e.g. Kordopatis et al. 2020, fig. 11). Therefore, determining the relative fractions of accreted and in situ retrograde stars in the MW requires detailed chemical analysis which is beyond the scope of this work.

The inner regions of the MW, which are difficult to study due to extinction and overcrowding, are currently being explored in greater detail. Queiroz et al. (2020) used Gaia DR2, along with chemistry from APOGEE DR16, to explore the bulge/bar region of the MW. They reported a highly eccentric, retrograde component within $1 \mathrm{kpc}$ of the Galactic Centre. While this inner retrograde component can indeed be generated by clumps sinking into the Galactic Centre, the bar, as we have shown, will also play a strong role in building up this population (see Fig. 3). We further demonstrated in Section 3.3 that bar-driven retrograde stars remain within the bar radius, and will therefore be centrally concentrated (bottom two rows in Figs 5 and 6), and we found in Section 3.2 that bars and clumps contribute roughly equally to the retrograde fraction in the inner regions.

\subsection{Implications for disc galaxies in general}

We found that the retrograde populations generated by the bar and clumps in the models do not produce the counterrotating discs observed in disc galaxies such as NGC 4550 (Rix et al. 1992; Rubin et al. 1992) and NGC 7217 (Merrifield \& Kuijken 1994). Indeed, we have shown in Section 3.4 that the retrograde stars more closely resemble a heated population in terms of their kinematics, rather than a rotationally supported disc. This can also be seen in Fig. 6, which illustrates how both clump and bar-driven retrograde stars are spheroidally distributed. The kinematically hot orbits combined with the paucity of retrograde stars in the outer disc region make it unlikely that such a retrograde population would have been detected in earlier surveys, which mainly used line-of-sight velocities to disentangle the counterrotating populations from the main disc. In one such study, Kuijken, Fisher \& Merrifield (1996) observed a sample of 28 S0 galaxies, including (at least) eight barred examples. They found that the galaxies in their sample must have a counterrotation mass fraction, $f_{\text {cr }} \lesssim 0.05$. In addition, they estimated that $\lesssim 10$ per cent of S0 galaxies have a significant retrograde population. However, in their study, only spectra in the flat portion of the rotation curves of their galaxies were considered, which means that the bulge/bar region was largely unexplored. Therefore, any retrograde population generated by the bar would remain undetected. In addition, any clump-driven retrograde stars in the outer disc would not be detected either, given how sparse they are in this region (see right column in Fig. 5). In more recent work, Zhu et al. (2018) constructed orbitsuperposition Schwarzschild models of galaxies observed in the 

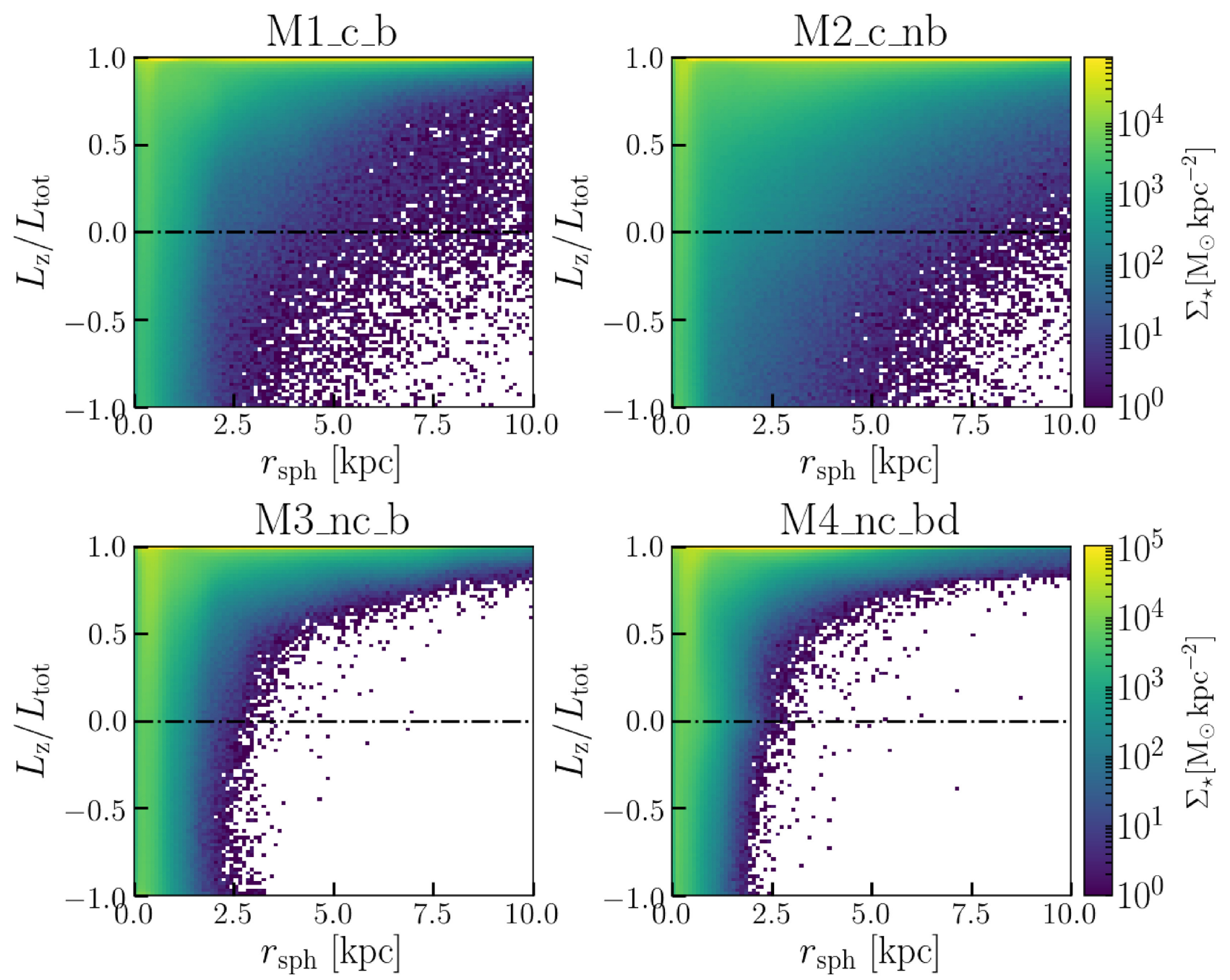

Figure 8. Density distribution in the space of $L_{\mathrm{z}} / L_{\mathrm{tot}}$ versus spherical radius $r_{\mathrm{sph}}$ at $10 \mathrm{Gyr}$ for the models. Stars with $L_{\mathrm{z}} / L_{\mathrm{tot}}$ close to unity are on in-plane orbits. On the other hand, stars with values of $L_{\mathrm{z}} / L_{\text {tot }}$ close to zero are on vertically heated orbits. The upper panels for the clumpy models show that the majority of retrograde stars in the outer regions (and to a lesser extent the inner regions) are on such vertically heated orbits.

CALIFA survey (Sánchez et al. 2012) and classified their orbits according to the circularity, $\lambda_{z}$. In general, they found that more massive galaxies tend to have higher fractions of both hot $\left(\left|\lambda_{z}\right|<\right.$ $0.25)$ and counterrotating $\left(\lambda_{z}<-0.25\right)$ orbits. However, from their sample, it is unclear whether there is any significant difference in the orbital configuration of barred and unbarred discs. To that end, it would be interesting to see if any future observational studies are able to confirm whether bars do indeed drive retrograde motion in the inner regions of disc galaxies, as the models predict.

Our findings have important implications with regard to the origin of stellar counterrotating discs. It is widely accepted that this phenomenon is produced by externally driven mechanisms such as the accretion of counterrotating gas which subsequently forms stars (e.g Coccato et al. 2011, 2013; Johnston et al. 2013; Pizzella et al. 2014,2018 ), a scenario which is supported by numerical simulations (Thakar \& Ryden 1996, 1998; Algorry et al. 2014), or possibly from merger events (Puerari \& Pfenniger 2001). However, there have also been efforts to link the phenomenon of counterrotation with internally driven mechanisms. For example, the 'wave patterns' observed in the velocity curves of barred S0 galaxies (Bettoni 1989; Bettoni \& Galletta 1997; Zeilinger et al. 2001) were interpreted by Wozniak
\& Pfenniger (1997) to be the result of a counterrotating population induced by the bar, and they further suggested that such a population might constitute 14-30 per cent of the stellar mass inside the bar's co-rotation resonance. Evans \& Collett (1994) also showed that it is possible for bar dissolution to generate a counterrotating population. In this scenario, stars on box orbits are able to escape the potential well to go on tube orbits as the potential becomes axisymmetric. In a non-rotating disc, there are as many stars on co-rotating boxy orbits as there are counterrotating. Therefore, half of the stars are scattered into counterrotating tube orbits. We have shown in Section 3.2 that bars are capable of generating a substantial retrograde population, in agreement with Wozniak \& Pfenniger (1997). However, the bardriven retrograde stars we find in the models are not counterrotating, and are restricted to the region of the bar. Furthermore, we showed that bar destruction is unable to produce counterrotating discs.

\section{SUMMARY}

By analysing four $\mathrm{N}$-body $+\mathrm{SPH}$ models of isolated disc galaxies, we investigated the role which stellar clumps and bars play in driving 
retrograde motion in disc stars. Our results can be summarized as follows:

(i) Both clumps and bars can generate quite a significant retrograde population (of order 10 per cent of the total stellar mass), with bars being slightly more efficient at producing retrograde stars. We find no evidence that bar dissolution drives additional retrograde motion (see Section 3.2).

(ii) Retrograde orbits generated by a bar remain confined to the inner regions inside the bar radius (even if the bar dissolves). Thus, any stars on retrograde motion found in the Solar neighbourhood cannot be attributed to the bar (see Section 3.3).

(iii) Retrograde orbits generated by clumps reach larger Galactocentric radii, and may be found in the Solar neighbourhood (see Section 3.3).

(iv) The retrograde orbits generated by clumps in the simulations represent of the stars in the mock Solar neighbourhood. This fraction is lower than that estimated in the MW (which includes both accreted and in situ stars), suggesting that the models do not overproduce 0.01-0.04 per cent retrograde stars (see Section 4.1).

(v) Neither bars nor clumps can produce rotationally supported counterrotating discs. Moreover, we find no evidence that bar destruction produces counterrotation (see Section 4.2).

\section{ACKNOWLEDGEMENTS}

The work reported on in this publication has been partially supported by COST Action CA18104: MW-Gaia. KF is partially funded by the the Tertiary Education Scholarships Scheme (TESS, Malta). KF thanks the Jeremiah Horrocks Institute for hospitality during a visit. VPD and LBS are supported by STFC Consolidated grant \#ST/R000786/1. The simulations in this paper were run at the High Performance Computing Facility of the University of Central Lancashire and at the DiRAC Shared Memory Processing system at the University of Cambridge, operated by the COSMOS Project at the Department of Applied Mathematics and Theoretical Physics on behalf of the STFC DiRAC HPC Facility (www.dirac.ac.uk). This equipment was funded by BIS National E-infrastructure capital grant ST/J005673/1, STFC capital grant ST/H008586/1, and STFC DiRAC Operations grant ST/K00333X/1. DiRAC is part of the National E-Infrastructure. JA acknowledges The World Academy of Sciences and the Chinese Academy of Sciences for the CAS-TWAS scholarship.

This research made use of PHOTUTILS, an ASTROPY package for detection and photometry of astronomical sources (Bradley et al. 2020).

We thank the anonymous referee for their constructive comments which helped to improve this paper.

\section{DATA AVAILABILITY}

The simulation data underlying this article are a mix of proprietary and available; some data may be shared upon reasonable request to V.P.D. (vpdebattista@gmail.com).

\section{REFERENCES}

Abadi M. G., Navarro J. F., Steinmetz M., Eke V. R., 2003, ApJ, 597, 21 Algorry D. G., Navarro J. F., Abadi M. G., Sales L. V., Steinmetz M., Piontek F., 2014, MNRAS, 437, 3596

Amarante J. A. S., Smith M. C., Boeche C., 2020a, MNRAS, 492, 3816

Amarante J. A. S., Beraldo e Silva L., Debattista V. P., Smith M. C., 2020b, ApJ, 891, L30
Athanassoula E., 2013, in Barroso J. F., Knapen J. H., eds, Bars and Secular Evolution in Disk Galaxies: Theoretical Input, Cambridge University Press, Cambridge. p. 305

Athanassoula E., Dehnen W., Lambert J. C., 2005, Highlights Astron., 13, 343

Belokurov V., Erkal D., Evans N. W., Koposov S. E., Deason A. J., 2018, MNRAS, 478, 611

Belokurov V., Sanders J. L., Fattahi A., Smith M. C., Deason A. J., Evans N. W., Grand R. J. J., 2020, MNRAS, 494, 3880

Beraldo e Silva L., Debattista V. P., Nidever D., Amarante J., Garver B. 2020a, MNRAS, 502, 260

Beraldo e Silva L., Debattista V. P., Khachaturyants T., Nidever D., 2020b, MNRAS, 492, 4716

Bettoni D., 1989, AJ, 97, 79

Bettoni D., Galletta G., 1997, A\&AS, 124, 61

Bournaud F., Combes F., Semelin B., 2005, MNRAS, 364, L18

Bournaud F., Elmegreen B. G., Martig M., 2009, ApJ, 707, L1

Bovy J., Rix H.-W., Liu C., Hogg D. W., Beers T. C., Lee Y. S., 2012, ApJ, 753,148

Bradley L. et al., 2020, astropy/photutils: 1.0.1

Buta R., Combes F., 1996, Fund. Cosmic Phys., 17, 95

Cava A., Schaerer D., Richard J., Pérez-González P. G., Dessauges-Zavadsky M., Mayer L., Tamburello V., 2018, Nat. Astron., 2, 76

Ceverino D., Dekel A., Bournaud F., 2010, MNRAS, 404, 2151

Chiappini C., Matteucci F., Romano D., 2001, ApJ, 554, 1044

Chiba R., Friske J. K. S., Schönrich R., 2021, MNRAS, 500, 4710

Clarke A. J. et al., 2019, MNRAS, 484, 3476

Coccato L., Morelli L., Corsini E. M., Buson L., Pizzella A., Vergani D., Bertola F., 2011, MNRAS, 412, L113

Coccato L., Morelli L., Pizzella A., Corsini E. M., Buson L. M., Dalla Bontà E., 2013, A\&A, 549, A3

Cole D. R., Debattista V. P., Erwin P., Earp S. W. F., Roškar R., 2014, MNRAS, 445,3352

Collett J. L., Dutta S. N., Evans N. W., 1997, MNRAS, 285, 49

Combes F., 2003, in Combes F., Barret D., Contini T., Pagani L., eds, SF2A2003: Semaine de l'Astrophysique Francaise, EDP Sciences, Paris. p. 243

Combes F., Gerin M., 1985, A\&A, 150, 327

Combes F., Sanders R. H., 1981, A\&A, 96, 164

Combes F., Debbasch F., Friedli D., Pfenniger D., 1990, A\&A, 233, 82

Cowie L. L., Hu E. M., Songaila A., 1995, AJ, 110, 1576

Das P., Hawkins K., Jofré P., 2020, MNRAS, 493, 5195

Debattista V. P., Sellwood J. A., 2000, ApJ, 543, 704

Debattista V. P., Carollo C. M., Mayer L., Moore B., 2004, ApJ, 604, L93

Debattista V. P., Mayer L., Carollo C. M., Moore B., Wadsley J., Quinn T., 2006, ApJ, 645, 209

Debattista V. P., Ness M., Gonzalez O. A., Freeman K., Zoccali M., Minniti D., 2017, MNRAS, 469, 1587

Dekel A., Sari R., Ceverino D., 2009, ApJ, 703, 785

Dessauges-Zavadsky M., Schaerer D., Cava A., Mayer L., Tamburello V., 2017, ApJ, 836, L22

Elmegreen B. G., 2009, in Jogee S., Marinova I., Hao L., Blanc G. A., eds, ASP Conf. Ser. Vol. 419, Galaxy Evolution: Emerging Insights and Future Challenges. Astron. Soc. Pac., San Francisco, p. 23

Elmegreen D. M., Elmegreen B. G., Ravindranath S., Coe D. A., 2007, ApJ, 658,763

Evans N. W., Collett J. L., 1994, ApJ, 420, L67

Förster Schreiber N. M. et al., 2011, ApJ, 739, 45

Frankel N., Sanders J., Rix H.-W., Ting Y.-S., Ness M., 2019, ApJ, 884, 99

Friedli D., Benz W., 1993, A\&A, 268, 65

Genzel R. et al., 2011, ApJ, 733, 101

Ghosh S., Debattista V. P., Khachaturyants T., 2020, preprint (arXiv:2009.02343)

Grand R. J. J. et al., 2020, MNRAS, 497, 1603

Guo Y., Giavalisco M., Ferguson H. C., Cassata P., Koekemoer A. M., 2012, ApJ, 757, 120

Guo Y. et al., 2015, ApJ, 800, 39 
Hasan H., Norman C., 1990, ApJ, 361, 69

Hawkins K., Jofré P., Masseron T., Gilmore G., 2015, MNRAS, 453, 758

Hayes C. R. et al., 2018, ApJ, 852, 49

Helmi A., Babusiaux C., Koppelman H. H., Massari D., Veljanoski J., Brown A. G. A., 2018, Nature, 563, 85

Hernquist L., Weinberg M. D., 1992, ApJ, 400, 80

Herpich J., Stinson G. S., Rix H. W., Martig M., Dutton A. A., 2017, MNRAS, 470,4941

Ho L. C., 2008, ARA\&A, 46, 475

Hohl F., 1971, ApJ, 168, 343

Hohl F., 1978, AJ, 83, 768

Johnston E. J., Merrifield M. R., Aragón-Salamanca A., Cappellari M., 2013, MNRAS, 428, 1296

Kalnajs A. J., 1973, Proc. Astron. Soc. Aust., 2, 174

Katkov I. Y., Sil'chenko O. K., Afanasiev V. L., 2013, ApJ, 769, 105

Kazantzidis S., Zentner A. R., Kravtsov A. V., Bullock J. S., Debattista V. P., 2009, ApJ, 700, 1896

Kordopatis G. et al., 2013, MNRAS, 436, 3231

Kordopatis G., Recio-Blanco A., Schultheis M., Hill V., 2020, A\&A, 643, A69

Kormendy J., 2013, Secular Evolution in Disk Galaxies, Cambridge University Press, Cambridge, p. 1

Kormendy J., Kennicutt, Robert C. J., 2004, ARA\&A, 42, 603

Kuijken K., Fisher D., Merrifield M. R., 1996, MNRAS, 283, 543

Martinez-Valpuesta I., Shlosman I., Heller C., 2006, ApJ, 637, 214

Merrifield M. R., Kuijken K., 1994, ApJ, 432, 575

Merritt D., Sellwood J. A., 1994, ApJ, 425, 551

Minchev I., Famaey B., Quillen A. C., Di Matteo P., Combes F., Vlajić M., Erwin P., Bland -Hawthorn J., 2012, A\&A, 548, A126

Moetazedian R., Just A., 2016, MNRAS, 459, 2905

Muñoz-Mateos J. C., Gil de Paz A., Boissier S., Zamorano J., Jarrett T., Gallego J., Madore B. F., 2007, ApJ, 658, 1006

Navarro J. F., Frenk C. S., White S. D. M., 1997, ApJ, 490, 493

Noguchi M., 1999, ApJ, 514, 77

Overzier R. A., Heckman T. M., Schiminovich D., Basu-Zych A., Gonçalves T., Martin D. C., Rich R. M., 2010, ApJ, 710, 979

Peebles P. J. E., 1969, ApJ, 155, 393

Pfenniger D., 1984, A\&A, 134, 373

Pfenniger D., Norman C., 1990, ApJ, 363, 391

Pizzella A., Morelli L., Corsini E. M., Dalla Bontà E., Coccato L., Sanjana G., 2014, A\&A, 570, A79

Pizzella A., Morelli L., Coccato L., Corsini E. M., Dalla Bontà E., Fabricius M., Saglia R. P., 2018, A\&A, 616, A22

Pontzen A., Roškar R., Stinson G. S., Woods R., Reed D. M., Coles J., Quinn T. R., 2013, pynbody: Astrophysics Simulation Analysis for Python.
Portaluri E. et al., 2017, MNRAS, 467, 1008

Posti L., Helmi A., Veljanoski J., Breddels M. A., 2018, A\&A, 615, A70

Puerari I., Pfenniger D., 2001, Ap\&SS, 276, 909

Queiroz A. B. A. et al., 2020, preprint (arXiv:2007.12915)

Raha N., Sellwood J. A., James R. A., Kahn F. D., 1991, Nature, 352, 411

Ravindranath S. et al., 2006, ApJ, 652, 963

Rix H.-W., Franx M., Fisher D., Illingworth G., 1992, ApJ, 400, L5

Roškar R., Debattista V. P., Quinn T. R., Stinson G. S., Wadsley J., 2008, ApJ, 684, L79

Rubin V. C., Graham J. A., Kenney J. D. P., 1992, ApJ, 394, L9

Sakamoto K., Okumura S. K., Ishizuki S., Scoville N. Z., 1999, ApJS, 124, 403

Sánchez S. F. et al., 2012, A\&A, 538, A8

Sellwood J. A., 1980, A\&A, 89, 296

Sellwood J. A., Athanassoula E., 1986, MNRAS, 221, 195

Sharma S., Hayden M. R., Bland-Hawthorn J., 2020, preprint (arXiv:2005.03646)

Shen J., Sellwood J. A., 2004, ApJ, 604, 614

Shen S., Wadsley J., Stinson G., 2010, MNRAS, 407, 1581

Sheth K., Vogel S. N., Regan M. W., Thornley M. D., Teuben P. J., 2005 , ApJ, 632, 217

Soto E. et al., 2017, ApJ, 837, 6

Sparke L. S., Sellwood J. A., 1987, MNRAS, 225, 653

Stinson G., Seth A., Katz N., Wadsley J., Governato F., Quinn T., 2006, MNRAS, 373, 1074

Swinbank A. M. et al., 2010, MNRAS, 405, 234

Thakar A. R., Ryden B. S., 1996, ApJ, 461, 55

Thakar A. R., Ryden B. S., 1998, ApJ, 506, 93

Toth G., Ostriker J. P., 1992, ApJ, 389, 5

Tremaine S., Weinberg M. D., 1984, MNRAS, 209, 729

Valenzuela O., Klypin A., 2003, MNRAS, 345, 406

van den Bergh S., Abraham R. G., Ellis R. S., Tanvir N. R., Santiago B. X., Glazebrook K. G., 1996, AJ, 112, 359

Velazquez H., White S. D. M., 1999, MNRAS, 304, 254

Wadsley J., Stadel J., Quinn T., 2004, New Astron., 9, 137

Wadsley J. W., Keller B. W., Quinn T. R., 2017, MNRAS, 471, 2357

Weinberg M. D., 1985, MNRAS, 213, 451

White S. D. M., Frenk C. S., 1991, ApJ, 379, 52

Wozniak H., Pfenniger D., 1997, A\&A, 317, 14

Zeilinger W. W., Vega Beltrán J. C., Rozas M., Beckman J. E., Pizzella A., Corsini E. M., Bertola F., 2001, Ap\&SS, 276, 643

Zhu L. et al., 2018, Nat. Astron., 2, 233

This paper has been typeset from a $\mathrm{T}_{\mathrm{E}} \mathrm{X} / \mathrm{L} \mathrm{T}_{\mathrm{E}} \mathrm{X}$ file prepared by the author. 